\section{El rol del vocabulario en la resolución de problemas} aritméticos: Un metaanálisis
Agosto 2021, Vol. 13, №2, 24-37

revistas.unc.edu.ar/inde x.php/racc

\author{
Ortiz, Sofía Soledad *,a , Sterpin, Lucas Federico a, Barreyro, Juan Pablo a,b y Formoso, Jesica a,b
}

Artículo Original

\section{Resumen}

El vocabulario se refiere al conocimiento acerca de las palabras que maneja y conoce una persona, y juega un rol importante en el desarrollo y la ejecución de gran cantidad de habilidades cognitivas. El objetivo de este estudio fue evaluar el tamaño del efecto del vocabulario en ambas modalidades, profundidad y amplitud, en las habilidades de resolución de problemas aritméticos. Con este propósito se realizó un meta-análisis sobre 16 estudios relacionados al tema. Se incluyeron estudios posteriores al año 2000, con niños entre 3 y 13 años y sin problemas neurológicos. Los resultados obtenidos mostraron un efecto moderado y significativo del vocabulario, tanto con respecto a la amplitud como a la profundidad del mismo, sobre la resolución de problemas aritméticos de tipo lingüístico. Los resultados también muestran un índice elevado de heterogeneidad, que puede ser explicado por las inconsistencias a la hora de evaluar los constructos.
Abstract

\begin{abstract}
The role of vocabulary in the resolution of arithmetic problems: A meta-analysis. Vocabulary refers to the knowledge about words that a person uses and understands. It plays a preponderated role in the development and execution of many cognitive abilities. The aim of this study was to evaluate the effect size of vocabulary in both of its modalities, breadth and depth, regarding the ability to solve arithmetic word problems. For this purpose, we conducted a meta-analysis on 16 studies related to the subject. We included studies done after 2000, with children aged 3 to 13 and without neurological problems. Our results showed a moderate but statistically significant effect of vocabulary on the ability to solve arithmetic word problems, without difference between its modalities. Results also show a high heterogeneity index that can be explained due to inconsistencies in the way the constructs are
\end{abstract} measured.

Keywords:

vocabulary, arithmetic problems, meta-analysis, children

vocabulario, problemas aritméticos, metaanálisis, niños

Recibido el 21 de diciembre de 2019; Aceptado el 1 de agosto de 2020

Editaron este artículo: Angel Elgier, Paula Abate, Nadia Justel y María Victoria Ortiz.

\title{
Introducción
}

El término problemas aritméticos hace referencia a ejercicios que involucran operaciones numéricas presentados en forma de narración en lugar de notación matemática, por ejemplo, si María tenía seis caramelos y regaló dos, ¿con cuántos caramelos se quedó? (Fuchs et al., 2006). Uno de sus objetivos principales es ayudar a los niños a concretizar conceptos matemáticos abstractos a través de emplazarlos en situaciones cotidianas, por lo que este tipo de problemas son un eslabón esencial en la enseñanza de las matemáticas, sobre todo durante los primeros años de la educación formal (Thevenot, 2017).

Esta forma particular de presentar conceptos matemáticos hace que poder resolverlos de forma efectiva, no sólo dependa del conocimiento que el niño tenga de las operaciones aritméticas, sino también de su capacidad para comprender el texto del problema, lo que permite pensar que otras capacidades o procesos cognitivos, más allá de los vinculados con el procesamiento numérico, pueden estar involucrados en su resolución y, entre ellos, aquellos de orden lingüístico, como el vocabulario.

\footnotetext{
a Universidad de Buenos Aires. Facultad de Psicología. Instituto de Investigaciones. Buenos Aires, Argentina.

${ }^{b}$ CONICET. Buenos Aires, Argentina.

*Enviar correspondencia a: Ortiz, S. E-mail: sofiaortiz628@gmail.com
}

Citar este artículo como: Ortiz, S. S., Sterpin, L. F., Barreyro, J. P. \& Formoso, J. (2021). El rol del vocabulario en la resolución de problemas aritméticos: Un metaanálisis. Revista Argentina de Ciencias del Comportamiento, 13(2), 24-37 
Para resolver un problema aritmético, el niño debe poder identificar las cantidades incluidas en el mismo, reconocer y recuperar de la memoria de largo plazo el algoritmo necesario para resolver la tarea, aplicarlo y monitorear el resultado (Geary, Hoard, Byrd-Craven, \& DeSoto, 2004; Groen \& Resnick, 1977; Ostad, 2000). Involucra habilidades como el conteo, la estimación de cantidades, el procesamiento de información numérica simbólica (ya sea etiquetas verbales o numerales arábigos), muchas de las cuales se automatizan durante la infancia, volviendo las estrategias de resolución más flexibles y precisas (Jordan, Hanich, \& Kaplan, 2003). Alcanzar el resultado correcto depende, además, de la construcción de una representación coherente del problema que describa las relaciones entre los componentes involucrados, y entre estos y el conocimiento previo del niño y de su traducción a un modelo matemático (Johnson-Laird, 2006; Múñez, Orrantia, \& Rosales, 2013; Thevenot, 2010).

El conocimiento previo general tiene un rol importante en el desarrollo y la ejecución de gran cantidad de habilidades cognitivas. Una de las maneras de evaluarlo es a través del vocabulario. El mismo es definido como el conocimiento acerca de las palabras que maneja y conoce una persona. Las palabras se almacenan en un diccionario mental en sus distintas manifestaciones: fonológica, ortográfica, sintáctica, semántica y morfológica (Emmorey \& Fromkin, 1992).

De acuerdo con Perfetti (Perfetti \& Hart, 2002), la calidad de la representación del léxico formada por el individuo será vital para la comprensión $y$, por consiguiente, para la comprensión de los problemas aritméticos. EI autor indica que cada palabra contiene tres constituyentes -el ortográfico, el fonológico y el semántico- y que la falta de calidad en alguno de estos daría como resultado posibles errores en la comprensión. Hoy en día, diferentes investigaciones muestran que el vocabulario se encuentra estrechamente relacionado con medidas de lectura (Cain \& Oakhill, 2011; Sesma, Mahone, Levine, Eason, \& Cutting, 2009) y comprensión de textos (Strasser \& del Río, 2014). En el área de la psicología del lenguaje, se sostiene que la buena calidad del mismo (alta precisión y flexibilidad dentro de la representación de palabras) ayuda a la recuperación de significados, a la resolución de ambigüedades, reduce la carga cognitiva y facilita la integración de palabras individuales en la representación del texto (Strasser \& del Río, 2014). La calidad de las representaciones que hacen al vocabulario se determina no sólo por el conocimiento lingüístico, sino también por su estabilidad (recuerdo consistente y confiable), su sincronicidad de activación (integración con el resto de las representaciones de conocimiento previo) y su velocidad de acceso (Gomes-Koban, Simpson, Valle, \& Defior, 2017).

Oakhill, Cain y McCarthy (2015) introducen los conceptos de amplitud y profundidad del vocabulario, para estudiar su rol en la comprensión de textos. La amplitud refiere a la cantidad o al número de palabras conocidas por un individuo, mientras que la profundidad refiere al conocimiento que se tenga de las mismas en términos de su significado, posibles usos y relaciones conceptuales (Oakhill et al., 2015). La amplitud se evalúa a partir de pruebas que implican la denominación o reconocimiento de imágenes, objetos y palabras, mientras que la profundidad se evalúa a partir de pruebas que requieren la definición de una palabra, ya sea a partir de su recuerdo o su reconocimiento. Si bien la amplitud y la profundidad han mostrado ser muy importantes en la comprensión, también se ha observado que muestran efectos distintos, la profundidad muestra un efecto de mayor intensidad que la amplitud sobre la comprensión, mientras que esta última muestra un efecto más relevante en la adquisición de la lectura (Oakhill \& Cain, 2012; Strasser \& del Río, 2014).

La relación entre el vocabulario y la resolución de problemas aritméticos está documentada. Sin embargo, los estudios que la describen difieren en cuanto a las medidas utilizadas para evaluar el conocimiento que tienen los niños de las palabras (Gilbert \& Fuchs, 2017; Purpura \& Ganley, 2014). Debido a que, cuando se busca resolver un problema aritmético se construye un modelo de situación de la descripción del mismo que implica la identificación de los componentes relevantes de la narración y las relaciones entre ellos, sería esperable que no bastase con el reconocimiento de las etiquetas de las palabras, sino que hiciera falta un conocimiento mayor del significado de las mismas. Es decir que al igual que en el caso de la comprensión de un texto general, la incidencia de la profundidad de vocabulario sobre la habilidad 
para resolver problemas de este tipo debería ser mayor que la de la amplitud. Es así que el objetivo del siguiente metaanálisis es estudiar posibles diferencias en el tamaño del efecto de la profundidad y la amplitud de vocabulario sobre la resolución de problemas aritméticos en niños en edad escolar.

\section{Método}

Se realizó una búsqueda en la literatura publicada para identificar estudios en los que se evaluó el vocabulario a partir de diferentes pruebas en niños de 3 a 13 años sin alteraciones. Se realizó la búsqueda en Pubmed, SciELO, PsycINFO, Redalyc y APA search, desde el primero de enero de 2000 hasta el 28 de octubre de 2019. Se utilizaron combinaciones de términos específicos tales como vocabulario, habilidad verbal, conocimiento previo, tanto en español como sus términos en inglés (vocabulary, verbal ability y prior knowledge) y términos vinculados a la resolución de problemas aritméticos tales como problemas aritméticos, matemática, cálculo, problemas de historia y sus correspondientes nombres en inglés (arithmetic word problems, arithmetic problems, mathematical problems y math problems). También se examinaron los artículos citados de los artículos recuperados. Se limitó la búsqueda a estudios en idioma inglés y español en revistas revisadas por pares.

\section{Selección de estudios}

Se utilizaron varios criterios de inclusión y exclusión para identificar artículos relevantes. Los criterios se refieren principalmente a la selección de participantes y se tuvo en cuenta el tipo de problema aritmético y de vocabulario.

Los artículos seleccionados tenían que:

(1) incluir niños sin alteraciones del desarrollo, del aprendizaje, psiquiátricas o neuropsicológicas de entre los 3 y los 13 años, tampoco se incluyeron niños con hipoacusia o alteraciones visuales, ni muestras con niños de nivel socioeconómico bajo.

(2) incluir problemas aritméticos presentados de forma oral o escrita que estuvieran basados en lenguaje, o fueran problemas de historias. Los problemas podían ser confeccionados por los autores, como pruebas ad-hoc, o ser parte de pruebas estandarizadas que evalúen habilidades matemáticas.

(3) incluir tareas o pruebas de vocabulario que podían ser presentadas de forma oral o escrita, y que evaluaran las dimensiones de amplitud o profundidad del vocabulario.

Un resumen del proceso de búsqueda bibliográfica puede encontrarse en el diagrama de la Figura 1.

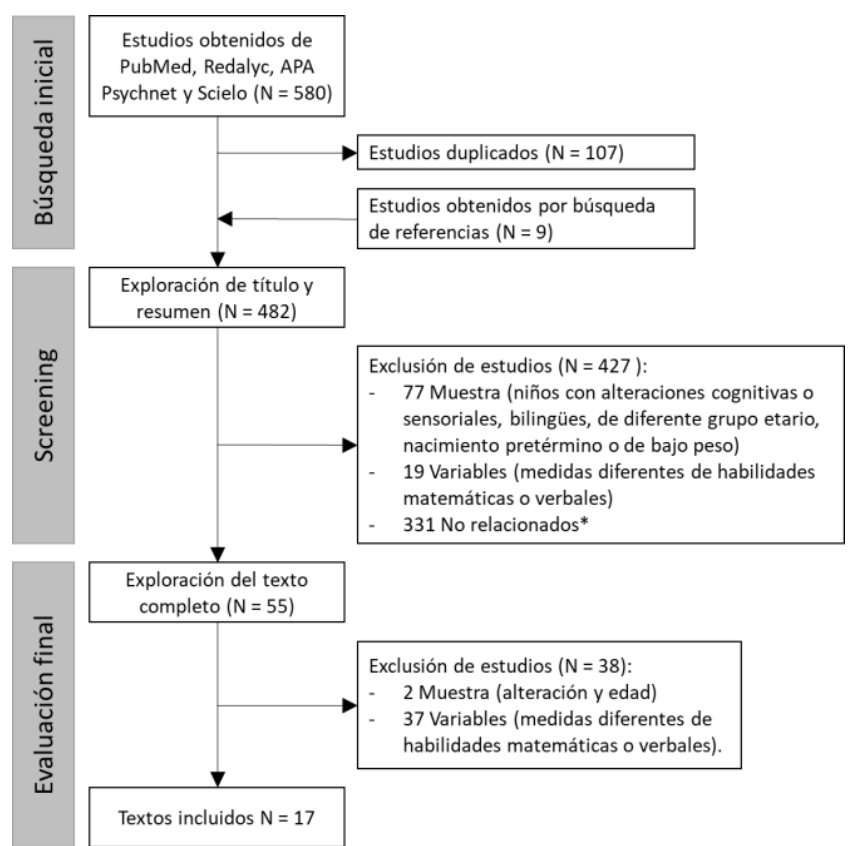

Figura 1. Diagrama de flujo del metaanálisis para la selección de estudios.

Nota: *Se consideraron como "no relacionados" aquellos artículos donde se combinan diferencias en la población objetivo y en las habilidades matemáticas y/o lingüísticas registradas, así como aquellos que se centran en el estudio de habilidades cognitivas no vinculadas a los objetivos de este estudio.

Este procedimiento nos permitió encontrar 17 artículos, los cuales se describen a continuación. Un resumen de los mismos puede encontrarse en la Tabla 1.

Fuchs et al. (2006) buscaron identificar características cognitivas vinculadas al desarrollo de las matemáticas en niños de 6 y 7 años de edad. Para ello, obtuvieron una serie de medidas de habilidades matemáticas, entre ellas la resolución de problemas aritméticos, y de habilidades cognitivas de dominio general como velocidad de procesamiento, memoria de trabajo y lenguaje. Para evaluar la resolución de problemas aritméticos utilizaron la tarea propuesta por Jordan y Hanich (2000). En ella los niños debían resolver 14 problemas de digito simple, algunos con resultados desconocido ("Sue tenía 5 peniques. Luego Mike le dio 2 peniques más. ¿Cuántos peniques tiene Sue ahora?") y otros con uno de 
los operandos faltante ("Karen tenía algunos peniques. Luego Matt le dio otros 4 peniques. Ahora Karen tiene 6 peniques. ¿Cuántos tenía al principio?'). Tanto la presentación como la respuesta eran en formato oral, aunque se les permitía a los niños tener con ellos la versión escrita. Como medida de lenguaje utilizaron el subtest de vocabulario de la Escala abreviada de inteligencia Wechsler (WASI: Wechsler, 1999), la que cuenta con 31 estímulos que se presentan al niño de forma oral y que él debe definir. Las respuestas se puntúan en base al nivel de abstracción de la misma. Los autores realizaron análisis de correlaciones bivariadas entre las variables objetivo $y$, específicamente para problemas aritméticos, encontraron asociaciones con memoria de trabajo, velocidad de procesamiento, procesamiento fonológico y lenguaje. Éstas últimas, de intensidad media. Utilizando esas mismas pruebas pero con una muestra de menor tamaño, Seethaler, Fuchs, L., Fuchs, y Compton (2012) obtuvieron una asociación significativa con un tamaño del efecto alto. Fuchs et al. (2005), en cambio, evaluaron problemas aritméticos presentados y resueltos oralmente, tomados del subtest de problemas aplicados de la escala Woodcock-Johnson III (Woodcock, McGrew, \& Mather, 2001), y si bien encontraron una asociación significativa con vocabulario, la magnitud del efecto es despreciable. Es posible que la significación esté asociada al tamaño de la muestra, siendo esta de 272 niños.

Fuchs et al. (2010a) tuvieron como objetivo examinar la relación de la cognición numérica básica y habilidades de dominio general con cálculo y problemas aritméticos en alumnos de primer grado. Al igual que en el estudio anterior, utilizaron los problemas de Jordan y Hanich (2000) pero evaluaron vocabulario utilizando la escala Woodcock Diagnostic Reading Battery (Woodcock, 1997), la cual presenta oraciones que plantean analogías y asociaciones con una palabra faltante que debe ser completada por el niño. El estudio mostró una asociación de intensidad alta. Los autores repitieron este mismo estudio (Fuchs et al., 2010b) tomando las mismas medidas a principio y final del año académico, y clasificando los problemas según tipo de operación mental requerida y en el análisis de correlaciones bivariadas encontraron asociaciones de intensidad media y alta.
Formoso, Injoque-Ricle, Jacubovich, y Barreyro (2017) tuvieron como objetivo estudiar la relación entre distintas habilidades cognitivas de dominio general con el cálculo mental en niños de 6 años. Para ello administraron una prueba con 6 adiciones y 6 sustracciones simples, con 2 y 3 operandos, siendo 18 el resultado más grande en las sumas y el operando más grande en las restas. Como medida de lenguaje se utilizó el subtest de vocabulario de la escala de inteligencia WISC-III (Wechsler, 1994), en el cual se presenta al niño una serie de palabras que él debe definir. En el estudio no se encontró una correlación significativa entre ambas variables. Männamaa, Kikas, Peets, y Palu (2012) presentaron cuatro problemas aritméticos ajustados al nivel educativo de los participantes (niños de 8 a 10 años) sin especificar las características de los mismos. Al igual que en el estudio anterior, se utilizó una prueba de definición de conceptos (The defining test: Kikas, Männamaa, Kumari, \& Ulst, 2008). En el estudio se describió una asociación de intensidad alta entre ambas variables.

Pina, Fuentes, Castillo, y Diamantopoulou (2014) estudiaron la influencia de la memoria de trabajo, la habilidad verbal y el status socioeconómico sobre las habilidades matemáticas en niños de 12 años. Los autores evaluaron problemas utilizando la versión española del subtest Aritmética de la batería de inteligencia WISC-IV (Wechsler, 2012). Se presentó a los niños un máximo de 34 problemas de dificultad creciente y se les pidió que indicaran que tipo de operación era necesaria para resolverlo (adición, sustracción, multiplicación o división). Para evaluar habilidad verbal utilizaron la prueba de acertijos de la versión española de la batería K-ABC (Batería de evaluación de Kaufman para niños - Kaufman, A. \& Kaufman, 1983), la cual evalúa la capacidad de los niños de inferir los nombres de conceptos concretos y abstractos a partir de una serie de pistas. En el estudio se describió una asociación de intensidad media entre las variables.

Kleemans, Segers, y Verhoeven (2018) evaluaron habilidad lingüística y su impacto en habilidades matemáticas en niños de 5to grado. Evaluaron vocabulario utilizando 25 palabras que los participantes debían identificar seleccionando la definición correcta entre varias opciones y problemas aritméticos presentados por escrito y resueltos con papel y lápiz, incluyendo las cuatro 
operaciones. En el estudio se encontró una asociación de intensidad baja entre las variables. De Smedt, Taylor, Archibald, y Ansari (2010) buscaron analizar cómo se relaciona el lenguaje con las diferencias individuales en habilidades aritméticas. Los autores diseñaron para el estudio problemas de dígito simple, excluyendo aquellos de empate (por ejemplo, $5+5$ ) y aquellos con 0 o 1 como operandos o resultados, con dos niveles de dificultad. Aplicaron esta tarea a niños de entre 9 y 11 años y la presentación fue por escrito, en computadora, y se utilizó el teclado para ingresar la respuesta. Realizaron correlaciones bivariadas y solo encontraron asociación de intensidad media entre vocabulario y los problemas de menor complejidad. Sin embargo, el tamaño del efecto fue medio en el caso de los problemas de mayor complejidad, por lo que es posible que con una muestra de mayor tamaño la asociación fuese significativa.

Purpura y Ganley (2014) y Hornburg, Schmitt, y Purpura (2018) se centraron en la relación entre lenguaje y habilidades matemáticas en niños de 3 a 5 años, 3 a 6 años y 4 años, respectivamente. Ambos estudios evaluaron la habilidad para resolver tres problemas de adición y cuatro de sustracción en formato oral, tanto para la presentación como para la respuesta, y utilizaron dos pruebas distintas de denominación como medida de lenguaje (EOWPVT: Brownell, 2000; CELF:P2: Wiig, Secord, \& Semel, 2004). En ambos estudios se encontraron asociaciones entre la medida de vocabulario y problemas aritméticos de intensidad alta y media-alta. Purpura, Hume, Sims, y Lonigan (2011) retomaron dichos estudios pero utilizando un test de vocabulario expresivo (TOPEL: Lonigan, Wagner, Torgesen, \& Rashotte, 2007) y obtuvieron resultados similares.

Blair, Ursache, Greenberg, y Vernon-Feagans (2015) buscaron analizar el efecto de la autorregulación sobre las matemáticas y la lectura en niños de 4 a 8 años. Evaluaron problemas aritméticos presentados y resueltos oralmente, tomados del subtest de problemas aplicados de la escala Woodcock-Johnson III (Woodcock et al., 2001) y vocabulario con una tarea de denominación (Peabody Picture Vocabulary Test, Dunn, L. \& Dunn, 2007) y encontraron asociaciones entre ambas de intensidad media. Si bien vocabulario fue incluido como una covariable, los autores analizaron las asociaciones bivariadas entre todas las variables incluidas.

Gilbert y Fuchs (2017) tuvieron como objetivo analizar la asociación entre cálculo y problemas aritméticos en niños de 7 y 8 años. Para ello plantearon un modelo de regresión incluyendo lenguaje como covariable. Los datos obtenidos en el presente trabajo se extrajeron de las correlaciones bivariadas descriptas en el estudio. Los autores tomaron como medidas de lenguaje el Woodcock Diagnostic Reading Battery (Woodcock, 1997) y como medida de problemas aritméticos el subtest de resolución de problemas de KeyMath-R (KM-R; Connolly, 1998), el cual incluye situaciones problemáticas incluyendo operaciones de suma, resta, multiplicación y división. Administraron las pruebas en tres ocasiones distintas y en todos los casos obtuvieron asociaciones significativas de intensidad media y alta.

Cowan y Powell (2014) estudiaron la asociación entre habilidades de dominio general y cognición numérica con habilidades matemáticas en niños de 8 a 10 años. Evaluaron vocabulario con una tarea de denominación (BPVS II, Dunn, L., Dunn, Whetton, \& Burley, 1997) y utilizaron problemas presentados de forma verbal y acompañado por imágenes tomados del Wechsler Individual Achievement Test (WIAT-II UK, Wechsler, 2005), detectando una asociación de intensidad alta. Con igual objetivo, pero en niños de 12 años, Cirino, Tolar, Fuchs, y Huston-Warren (2016) utilizaron el subtest de la batería WASI, y 25 problemas aritméticos utilizando fracciones y obtuvieron una asociación de intensidad alta.

A pesar del bajo número de estudios encontrados, no surgen preocupaciones particulares en la literatura sobre el tamaño de la muestra para un metaanálisis (Green \& Higgins, 2006), por lo que se procedió con el estudio

\section{Análisis de datos}

La búsqueda sistemática arrojó 17 artículos que cumplieron con los criterios de inclusión y exclusión descriptos previamente, de los cuales se obtuvieron 27 medidas de efecto (Figura 2). Los mismos fueron agrupados dependiendo de si evaluaron vocabulario según su amplitud o su profundidad. Para llevar a cabo el análisis, se utilizó el coeficiente de correlación productomomento $(r)$ como estimación del tamaño del efecto. Siendo que, a excepción de uno de los estudios, este se encontró reportado en todos los. 
Ortiz, S. S., Sterpin, L. F., Barreyro, J. P. y Formoso, J. / RACC, 2021, Vol. 13, N², 24-37

Tabla 1.

Características de los estudios incluidos en los análisis

\begin{tabular}{|c|c|c|c|c|c|c|c|}
\hline Autores & $N$ & Edad & $\begin{array}{l}\text { Tareas que evalúan } \\
\text { problemas aritméticos }\end{array}$ & Presentación & Resolución & $\begin{array}{l}\text { Tareas que evalúan } \\
\text { vocabulario }\end{array}$ & $\begin{array}{l}\text { Medida de } \\
\text { vocabulario }\end{array}$ \\
\hline Blair et al., (2015) & 1292 & $4-8$ & $\begin{array}{l}\text { Woodcock-Johnson III (WJ III) } \\
\text { Applied Problems subtest } \\
\text { (Woodcock et al., 2001) }\end{array}$ & Oral & Oral & $\begin{array}{l}\text { Peabody Picture } \\
\text { Vocabulary Test, 4th Ed. } \\
\text { Dunn, L. \& Dunn, (2007) }\end{array}$ & Amplitud \\
\hline Cirino et al., (2016) & 162 & $11-13$ & $\begin{array}{l}\text { Brown and Quinn Fraction } \\
\text { Competency Test (Brown \& } \\
\text { Quinn, 2007a, 2007b) }\end{array}$ & Leído & Escrito & $\begin{array}{l}\text { Wechsler Abbreviated } \\
\text { Scales of Intelligence } \\
\text { (WASI) Vocabulary subtest } \\
\text { (Wechsler, 1999) }\end{array}$ & Profundidad \\
\hline Cowan y Powell (2014) & 258 & $8-10$ & $\begin{array}{l}\text { Mathematical Reasoning } \\
\text { subtest del Wechsler } \\
\text { Individual Achievement Test. } \\
\text { 2nd UK Ed. (Wechsler, 2005) }\end{array}$ & Oral & Oral & $\begin{array}{c}\text { British Picture Vocabulary } \\
\text { Scale (BPVS II) (Dunn et } \\
\text { al., 1997) }\end{array}$ & Amplitud \\
\hline De Smedt et al. (2010) & 37 & $9-11$ & $\begin{array}{c}\text { 'Set Standard' de digito- } \\
\text { simple de problemas } \\
\text { aritméticos (LeFevre et al., } \\
\text { 1996) }\end{array}$ & Escritas & Teclas PC & $\begin{array}{c}\text { Comprehensive Test of } \\
\text { Phonological Processing, } \\
\text { Standardized Phoneme } \\
\text { Elision subtest (Wagner et } \\
\text { al., 1999) }\end{array}$ & Amplitud \\
\hline Formoso et al. (2017) & 70 & 6 & $\begin{array}{c}6 \text { ítems de sumas simples con } \\
2 \text { y } 3 \text { sumandos }+6 \text { de restas } \\
\text { simples con } 2 \text { y } 3 \text { sustraendos } \\
\text { (ad hoc). }\end{array}$ & Oral & Oral & $\begin{array}{l}\text { Subtest de vocabulario } \\
\text { WISC III (Wechsler, 1994) }\end{array}$ & Profundidad \\
\hline
\end{tabular}


Ortiz, S. S., Sterpin, L. F., Barreyro, J. P. y Formoso, J. / RACC, 2021, Vol. 13, N², 24-37

Continuación...

Fuchs et al. (2005)

Fuchs et al. (2010a)

$6-7$

Story Problems (Jordan \&

Hanich, 2000)

Escrita

Escrita

Applied Problems subtest

(Woodcock et al., 2001)

Oral y

escrita

Oral y

escrita

Story Problems (Jordan \&

Hanich, 2000)

Escrita

Escrita

Fuchs et al. (2010b)

$280 \quad 6-8$

$3128-9$

Story Problems (Jordan \&

Hanich, 2000)

Fuchs et al., (2006)

Gilbert y Fuchs (2017)

328

$7-8$

KeyMath-R Problem Solving

(Connolly, 1998).

3 items de sumas simples +4 de restas simples sin distractores.

Oral

Oral

Hornburg et al. (2018)

124

$3-6$

$167 \quad 10-12$
Speed Test for Arithmetic Fluency (De Vos, 2010)
Wechsler Abbreviated

Scales of Intelligence

(WASI) Vocabulary subtest

(Wechsler, 1999)

Wechsler Abbreviated

Scales of Intelligence

(WASI) Vocabulary subtest

(Wechsler, 1999)

Woodcock Diagnostic

Reading Battery, Escala de comprensión (Woodcock, 1997)

Wechsler Abbreviated

Scales of Intelligence

(WASI) Vocabulary subtest

(Wechsler, 1999)

Wide range Achievement

Test 3, Word Reading

subtest (Wilkinson, 1993)

Expressive Vocabulary

subtest del Clinical

Evaluation of Language

Fundamentals-Preschool-

Second Edition (Wiig et al., 2004)

Kleemans et al. (2018)
Escrito
54 items multiple-choice sobre ciencia y tecnología.
Profundidad

Profundidad

Amplitud

Profundidad

Profundidad

Amplitud
Profundidad 
Continuación...

Männamaa et al. (2012)

Pina et al. (2014)

Purpura y Ganley

(2014)

Purpura et al. (2011)

Seethaler et al. (2012)
723

$8-10$

102

$9-13$

199

$$
4-6
$$

$$
3-5
$$
acordes al nivel educativo de los participantes (ad hoc)

$\begin{array}{ll}\text { Escrito } & \text { Escrito } \\ \text { Oral } & \text { Oral } \\ \text { Oral } & \text { Oral } \\ \text { Oral } & \text { Oral } \\ \begin{array}{ll}\text { Oral y } \\ \text { escrito }\end{array} & \text { Escrito }\end{array}$
Applied Problems subtest (Woodcock et al., 2001)

\section{Story Problems (Jordan \&} Hanich, 2000) The Defining Test (Kikas et
al., 2008)

K-ABC. Subescala de adivinanzas (Kaufman, A. \& Kaufman, 1983)

The Expressive One-Word Picture Vocabulary Test. 3rd Ed. (Brownell, 2000)

Subtest de vocabulario de Test TOPEL (Lonigan et al., 2007) seleccionados, el mismo se extrajo directamente de la sección de resultados de los mismos. En Formoso et al. (2017) se reportó la significación de la correlación, pero no el coeficiente, por lo que se revisaron las bases originales para obtener el estimador.

En el caso de De Smedt et al. (2010) se incluyeron los coeficientes de correlación vinculados a la resolución de problemas aritméticos pequeños y grandes. Los autores se basaron para ello en la clasificación de Campbell y Xue (Campbell \& Xue, 2001), según la cual los problemas pequeños son aquellos cuya resolución depende mayormente de la recuperación de hechos aritméticos desde la memoria de largo plazo.

De Gilbert y Fuchs (2017) se extrajeron tres coeficientes de correlación de un estudio longitudinal correspondientes a las medidas obtenidas al principio y al final del $2^{\circ}$ grado y al final del $3^{\circ}$ grado.

Fuchs et al. (2010a) incluyeron tres tipos de problemas aritméticos basados en Jordan y Hanich (2000): a. dos cantidades se combinan para alcanzar un total; b. dos cantidades se comparan para encontrar una diferencia; c. una acción desencadena un cambio (aumento o disminución) de la cantidad inicial. Para cada uno de ellos se obtuvo una medida al inicio y otra al final del año escolar, resultando en un total de 6 coeficientes de correlación. Adicionalmente, los autores utilizaron ecuaciones estructurales para calcular puntajes basados en dos factores latentes, uno correspondiente a las medidas iniciales y uno a las finales, resultando 
en dos coeficientes de correlación adicionales.

El análisis inicial incluyó todas las medidas mencionadas previamente, a excepción de los coeficientes obtenidos a partir de los factores latentes en Fuchs et al. (2010a). En un segundo momento, se realizaron análisis de sensibilidad utilizando puntajes promediados, por un lado, de las distintas medidas de problemas aritméticos (problemas pequeños y grandes en De Smedt et al. [2010] y de combinar, comparar y cambiar en Fuchs et al. [2010a]) y por otro, de los distintos tiempos de evaluación en los estudios de corte longitudinal. Finalmente, se repitió el análisis tomando una medida única promediada de cada estudio.

Para obtener un estimador global de la asociación entre la resolución de problemas aritméticos y el vocabulario y su correspondiente intervalo de confianza, se utilizó el modelo de efectos aleatorios con el método de máxima verosimilitud restringida para estimar la varianza interestudio (Langan et al., 2019; Veroniki et al., 2016). Se seleccionó dicho modelo debido a que no es posible asumir que las distintas muestras pertenezcan a una única población homogénea (Borenstein, Hedges, Higgins, \& Rothstein, 2010). Se utilizó la transformación Z de Fisher sobre los coeficientes de los estudios individuales para obtener medidas ponderadas según el tamaño de la muestra. La proporción de varianza observada que se debe a heterogeneidad se calculó a través del índice $\mathrm{I}^{2}$.

Finalmente, se realizaron pruebas de hipótesis para evaluar posibles diferencias entre el tamaño del efecto en estudios que evaluaron amplitud y profundidad de vocabulario (test de Wald) y entre los modelos planteados para los análisis de sensibilidad (test de cociente de verosimilitud o likelihood ratio test)

\section{Resultados}

El $87.5 \%$ de los estudios fueron publicados entre 2010 y 2019, siendo los años con mayor publicación 2010 (3) y 2014 (4). Sólo el 25\% incluyeron niños mayores a 9 años en la muestra. El $62.5 \%$ evaluaron el vocabulario en términos de profundidad, de los cuales el $70 \%$ (43.5\% del total) utilizó la prueba de vocabulario de las baterías Wechsler.

En el diagrama de bosque o forest plot (Figura 2) pueden observarse los resultados obtenidos utilizando el modelo de efectos aleatorios con el método de máxima verosimilitud restringida para estimar la varianza interestudio sobre a la totalidad de las medidas extraídas (26). Se observó una correlación global promedio significativa de intensidad media entre vocabulario y resolución de problemas aritméticos $(r=.51$, IC $95 \%[.44, .57])$.

Los estimadores globales son similares si tomamos por separado los estudios que evalúan amplitud de vocabulario ( $r=.51$, IC 95\% [.43, .59]) y aquellos que evalúan profundidad $(r=.51$, IC $95 \%[.42, .60])$. Tanto agrupando todos los estudios, como diferenciando por tipo de medida de vocabulario, se observa un porcentaje alto de heterogeneidad interestudio $\left(\mathrm{I}^{2}\right.$ total $=86.5 \%$, IC 95\% [77.42 - 93.14], $\mathrm{I}^{2}$ Amplitud $=69.6 \% ; \mathrm{I}^{2}$ Profundidad $=89 \%$ ). La alta heterogeneidad indica que los efectos descriptos por los distintos estudios son muy diversos por lo que el coeficiente global es poco confiable y los resultados no son generalizables. La escasez de estudios disponibles no permite analizar otras variables como posibles moderadoras, como podrían serlo las diferentes edades, los instrumentos utilizados o el año de publicación. Por otro lado, la bibliografía sugiere que en metaanálisis realizados con pocos estudios el estadístico $\mathrm{I}^{2}$ tiende a subestimar la heterogeneidad (Von Hippel, 2015) por lo que se recomienda interpretar la misma a partir del intervalo de confianza al $95 \%$ y no a su estimación puntual. No se encontraron diferencias significativas en la asociación entre las variables entre amplitud y profundidad (Test de Wald $=.03$, $p=.86)$.

Para los análisis de sensibilidad se ajustaron tres modelos con los mismos criterios que en el análisis anterior, pero promediando los coeficientes extraídos de un mismo estudio (Tabla 2). En el primero se promediaron los coeficientes de distintas medidas de problemas aritméticos, en el segundo los coeficientes obtenidos de medidas realizadas en distintos tiempos (estudio longitudinal) y, finalmente, promediando todas las medidas pertenecientes al mismo trabajo. Los resultados no muestran diferencias relevantes entre los coeficientes obtenidos en uno y otro estudio. En todos los casos se observa que las correlaciones de los problemas de aritméticos son más altas con las medidas de amplitud que con las de profundidad, pero estas diferencias no son estadísticamente significativas. 


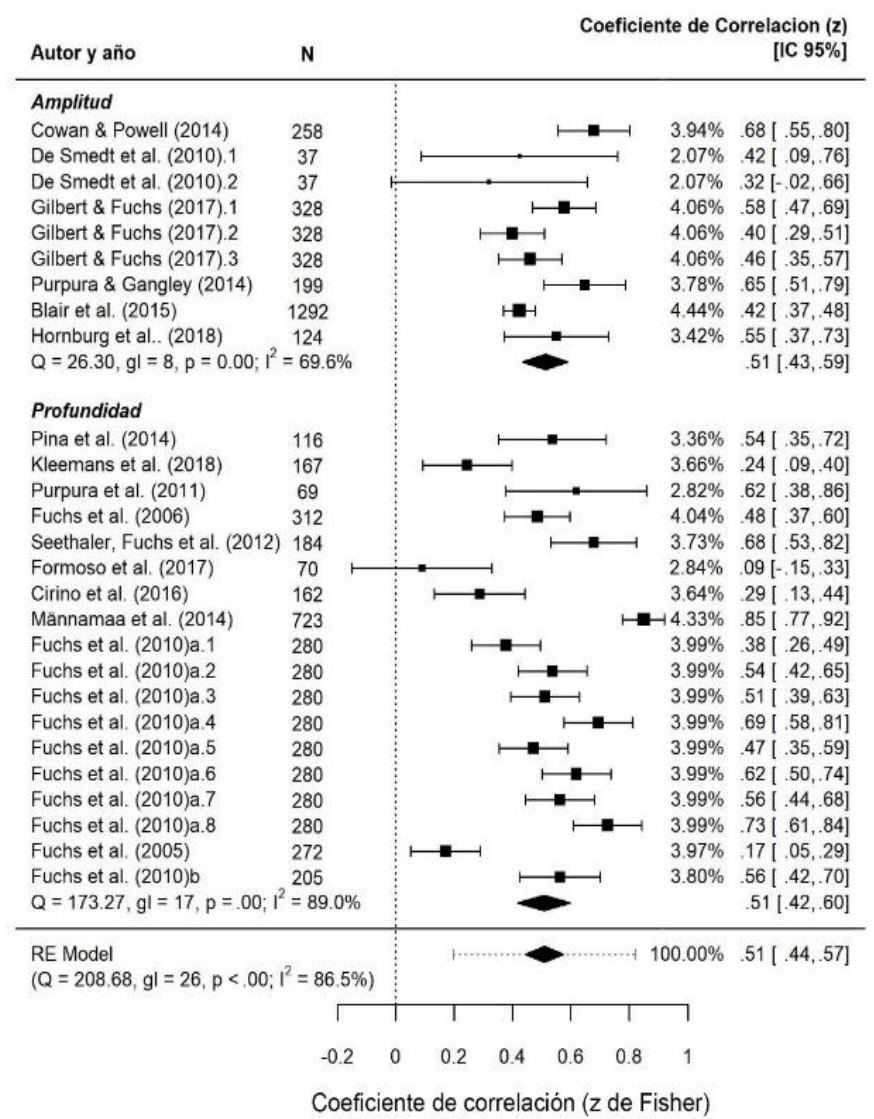

Figura 2. Forest plot del tamaño del efecto obtenido en los estudios según evaluaran amplitud o profundidad de vocabulario.

\section{Discusión}

En las últimas décadas la investigación en psicología del desarrollo, neurociencia y psicología cognitiva han identificado ciertas habilidades matemáticas básicas de aparición temprana como predictoras de habilidades más complejas de adquisición posterior (Inglis \& Gilmore, 2014; Odic, Libertus, Feigenson, \& Halberda, 2013; Park \& Brannon, 2013; Sasanguie, Defever, Maertens, \& Reynvoet, 2014; Starr, Libertus, \& Brannon, 2013). Una línea de investigación se ha centrado en la posibilidad de predecir el desarrollo atípico de la función a través de la evaluación de estas habilidades básicas, esto es, poder realizar diagnósticos de alteraciones de forma previa al ingreso del niño a la educación formal (Bugden \& Ansari, 2016; Mazzocco, Feigenson, \& Halberda, 2011). En este sentido, es importante destacar que las investigaciones muestran el rol del vocabulario en algunas de las habilidades matemáticas básicas, como es la resolución de problemas aritméticos
(Fuchs et al., 2010a; Fuchs et al., 2010b; Hornburg et al., 2018; Purpura \& Ganley, 2014).

En el presente metaanálisis se estudió el rol del vocabulario en la resolución de problemas aritméticos tales como problemas matemáticos, cálculo, problemas de historia, con especial énfasis en la magnitud de su efecto, teniendo en cuenta la diferencia entre amplitud y profundidad de vocabulario, empleada en el ámbito de la psicología del lenguaje (Oakhill et al., 2015; Perfetti \& Hart, 2002). Partiendo del supuesto que la resolución de un problema aritmético requiere de la construcción de una representación mental que describa los componentes involucrados, e integre esta información con la proveniente del conocimiento previo (Johnson-Laird, 2006; Thevenot, 2010), y reconociendo el rol preponderante que tiene la profundidad del vocabulario en la comprensión del texto (Oakhill \& Cain, 2012), se podría esperar que la profundidad del vocabulario mostrase una asociación de mayor intensidad con el desempeño frente a problemas aritméticos que la amplitud.

Para este fin se realizó una búsqueda de la bibliografía relevante en Pubmed, SciELO, PsycINFO, Redalyc y APA Psychnet, incluyendo términos tales como vocabulario, habilidad verbal, conocimiento previo, problemas aritméticos, matemática, cálculo y problemas de historia. Se incluyeron aquellos estudios realizados a partir del año 2000, con niños de 3 a 13 años sin alteraciones psicológicas 0 psiquiátricas, y publicados tanto en inglés como en español. Esta revisión arrojó un total de 16 artículos.

Los resultados mostraron un efecto significativo y de mediana intensidad del vocabulario sobre la resolución de problemas aritméticos $(r=.50)$. Los problemas aritméticos son aquellos que requieren una serie de pasos para su resolución y que son presentados en formato lingüístico (Fuchs et al., 2006). Los resultados indicaron que el vocabulario, referido al conocimiento de las palabras y su significado está asociado estrechamente en la resolución correcta de problemas aritméticos en niños.

Al analizar el efecto específico de la amplitud de vocabulario sobre la resolución de los problemas también se observó un efecto de mediana intensidad y significativa $(r=.51)$. Esto indica que la cantidad de palabras que un niño conoce o su correspondiente etiqueta léxica se asocia de manera directa en la resolución. 
Ortiz, S. S., Sterpin, L. F., Barreyro, J. P. y Formoso, J. / RACC, 2021, Vol. 13, №2, 24-37

Tabla 2.

Análisis de sensibilidad

Promediado de

\begin{tabular}{|c|c|c|c|c|c|c|}
\hline $\begin{array}{l}\text { Promediado de } \\
\text { coeficientes }\end{array}$ & $\begin{array}{l}\text { Medida de } \\
\text { vocabulario }\end{array}$ & $n$ & Estudios promediados & $r\left[\mathrm{IC}_{95 \%}\right]$ & |2\% & $p$ \\
\hline \multirow{3}{*}{ Sin promediar } & Todas & 26 & & $.50[.43 ; .57]$ & $88.66 \%$ & \multirow{3}{*}{.86} \\
\hline & Amplitud & 9 & De Smedt et al. (2010) & $.51[.43 ; .59]$ & $69.61 \%$ & \\
\hline & Profundidad & 17 & Fuchs et al. (2010a) & $.49[.39 ; .59]$ & $91.16 \%$ & \\
\hline \multirow{3}{*}{$\begin{array}{l}\text { Distintos tipos de } \\
\text { problemas aritméticos }\end{array}$} & Todas & 19 & & $.48[.39, .56]$ & $90.45 \%$ & \multirow{3}{*}{.44} \\
\hline & Amplitud & 8 & Gilbert \& Fuchs (2017) & $.52[.44, .60]$ & $72.66 \%$ & \\
\hline & Profundidad & 11 & Fuc & $.44[.30, .59]$ & $92.72 \%$ & \\
\hline \multirow{3}{*}{$\begin{array}{l}\text { Distintos tiempos de } \\
\text { medición }\end{array}$} & Todas & 19 & & $.47[.38, .56]$ & $90.07 \%$ & \multirow{3}{*}{.51} \\
\hline & Amplitud & 7 & & $.52[.49 ; .62]$ & $68.77 \%$ & \\
\hline & Profundidad & 12 & & $.45[.32 ; .58]$ & $92.20 \%$ & \\
\hline \multirow{3}{*}{$\begin{array}{l}\text { Pertenecientes a un } \\
\text { mismo estudio }\end{array}$} & Todas & 16 & & $.47[.37 ; .58]$ & $91.69 \%$ & \multirow{3}{*}{1} \\
\hline & Amplitud & 6 & $\begin{array}{l}\text { De Smedt et al. (2010) } \\
\text { Gilbert \& Fuchs (2017) }\end{array}$ & $.53[.43 ; .63]$ & $72.70 \%$ & \\
\hline & Profundidad & 10 & Fuchs et al. (2010a) & $.43[.28 ; .59]$ & $93.25 \%$ & \\
\hline
\end{tabular}

Nota. $n=$ cantidad de coeficientes individuales incluidos; $r$ = coeficiente de correlación de Pearson global; $\left.\right|^{2}=$ índice de heterogeneidad; Test de Wald para evaluar diferencias entre Amplitud y Profundidad. ${ }^{*}<.05,{ }^{* *}<.01$

En cuanto a la profundidad de vocabulario, que se refiere al conocimiento acerca del significado de las palabras y, por lo tanto, a la calidad de la representación léxica semántica, los resultados mostraron que el efecto también fue intensidad media y significativa $(r=.49)$.

Ambos resultados indicaron que en la resolución de problemas aritméticos ambas dimensiones del vocabulario son necesarias. Si bien en el área de la comprensión del texto, la representación del modelo de situación que el texto describe requiere del conocimiento de la profundidad del vocabulario con mayor incidencia en comparación a la amplitud, requerida para la lectura. Este resultado obtenido a partir de este metaanálisis indicaría que durante la construcción del modelo mental del problema se requiere de igual medida del conocimiento acerca de la etiqueta de la palabra (amplitud) y de la riqueza del significado de la misma (profundidad).

Los resultados también mostraron que las asociaciones encontradas presentaron un elevado nivel de heterogeneidad. Este nivel de heterogeneidad puede ser explicado en base a las diferentes medidas utilizadas por los estudios para evaluar los problemas aritméticos, la cantidad de ítems y las diferentes modalidades. Puesto que no existe un método unificado de evaluación de la capacidad de resolver problemas aritméticos, como sí a criterios más precisos para la evaluación de la amplitud y de la profundidad del vocabulario, existen variaciones en cuanto a la manera de evaluar los problemas, que pueden llevar a distintos niveles de dificultad en cada caso. Por ejemplo, en Purpura y Ganley (2014) y en Hornburg et al., (2018) se presentaron tres ítems de adición y cuatro de sustracción sobre contenidos que los niños podrían considerar atractivos y sin información distractora, otorgándoles un punto por cada respuesta correcta; mientras que en Fuchs et al. (2006) se utilizó una batería de catorce problemas cortos, con sumas y restas simples, y que abarcaban problemas de cambio, combinación, comparación y ecualización. Por su parte, Gilbert y Fuchs (2017) usaron la batería Problem Solving del KM$\mathrm{R}$, que incluye dieciocho problemas de dificultad incremental y que comprenden las cuatro operaciones básicas — suma, resta, multiplicación y división- y en los que algunos poseen una estrategia de resolución transparente.

A nivel general, los resultados indican que el vocabulario tanto en su modalidad vinculada a la amplitud como la de la profundidad tiene un rol preponderante en la resolución de problemas aritméticos de tipo lingüístico. Desde el punto de vista de la educación, el desarrollo del vocabulario no debe ser visto como una asignatura independiente (propio de la asignatura de Idioma - Lengua) sino articulada a otras y que, asimismo, incide en el desempeño de los niños en aspectos no socialmente vinculados, como es el de las Matemáticas.

Teniendo en cuenta lo planteado, futuros metaanálisis que tengan como propósito estudiar la resolución de problemas aritméticos deberían 
tener en cuenta la modalidad de problemas con la que se evalúa (oral o escrito, de desarrollo o elección múltiple de respuesta). Asimismo, considerar también la cantidad de ítems utilizados en las pruebas. Futuras investigaciones podrían centrarse en la confección de pruebas estandarizadas que evalúen la resolución de problemas aritméticos debido a la heterogeneidad de medidas encontradas.

\section{Referencias}

Blair, C., Ursache, A., Greenberg, M., \& VernonFeagans, L. (2015). Multiple aspects of selfregulation uniquely predict mathematics but not letter-word knowledge in the early elementary grades. Developmental Psychology, 51(4), 459472. doi: $10.1037 / \mathrm{a} 0038813$

Borenstein, M., Hedges, L. V., Higgins, J. P. T., \& Rothstein, H. R. (2010). A basic introduction to fixed-effect and random-effects models for metaanalysis. Research Synthesis Methods, 1(2), 97111. doi: $10.1002 / \mathrm{jrsm} .12$

Brown, G., \& Quinn, R. (2007a). Fraction proficiency and success in algebra: What does the research say? The Australian Mathematics Teacher, 63(3), 23-30. doi: 10.3316/informit.136647932230670

Brown, G., \& Quinn, R. (2007b). Investigating the relationship between fraction proficiency and success in algebra. The Australian Mathematics Teacher, 63(4), 8-15. doi: 10.3316/informit.137057857598350

Brownell, R. (2000). Expressive one-word picture vocabulary test manual. Novato, California: Plural Publishing Inc.

Bugden, S., \& Ansari, D. (2016). Probing the nature of deficits in the 'Approximate Number System' in children with persistent Developmental Dyscalculia. Developmental Science, 19(5), 817-833. doi: 10.1111/desc. 12324

Cain, K., \& Oakhill, J. (2011). Matthew Effects in Young Readers. Journal of Learning Disabilities, 44(5), 431-443. doi: 10.1177/0022219411410042

Campbell, J. I., \& Xue, Q. (2001). Cognitive arithmetic across cultures. Journal of Experimental Psychology. General, 130(2), 299-315. doi: 10.1037//0096-3445.130.2.299

Connolly, A. J. (1998). Keymath - Revised. Circle Pines, Minnesota: American Guidance Service.

Cirino, P. T., Tolar, T. D., Fuchs, L. S., \& HustonWarren, E. (2016). Cognitive and numerosity predictors of mathematical skills in middle school. Journal of Experimental Child Psychology, 145, 95-119. doi: 10.1016/j.jecp.2015.12.010

Cowan, R., \& Powell, D. (2014). The contributions of domain-general and numerical factors to third- grade arithmetic skills and mathematical learning disability. Journal of Educational Psychology, 106(1), 214-229. doi: 10.1037/a0034097

De Smedt, B., Taylor, J., Archibald, L., \& Ansari, D. (2010). How is phonological processing related to individual differences in children's arithmetic skills? Developmental Science, 13(3), 508-520. doi: 10.1111/j.1467-7687.2009.00897.x

De Vos, T. (2010). Tempotest Automatiseren [Speed Test for Arithmetic Fluency]. Amsterdam: Boom test uitgevers.

Dunn, L. M., \& Dunn, D. M. (2007). Peabody picture vocabuary test: Form B. Minneapolis, Minnesota: Pearson.

Dunn, L. M., Dunn, L. M., Whetton, C., \& Burley, J. (1997). British Picture Vocabulary Scale II. Windsor, England: NFER-Nelson.

Emmorey, K. D., \& Fromkin, V. A. (1992). El léxico mental. En F. Newmayer (Ed.), Panorama de la lingüística moderna de la Universidad de Cambridge. Tomo III: Aspectos psicológicos y biológicos (pp. 151-176). Madrid, España: Visor.

Formoso, J., Injoque-Ricle, I., Jacubovich, S., \& Barreyro, J. P. (2017). Cálculo mental en niños y su relación con habilidades cognitivas. Acta de Investigación Psicológica, 7(3), 2766-2774. doi: 10.1016/j.aipprr.2017.11.004

Fuchs, L. S., Compton, D. L., Fuchs, D., Paulsen, K., Bryant, J. D., \& Hamlett, C. L. (2005). The prevention, identification, and cognitive determinants of math difficulty. Journal of Educational Psychology, 97(3), 493-513. doi: 10.1037/0022-0663.97.3.493

Fuchs, L. S., Fuchs, D., Compton, D. L., Powell, S. R., Seethaler, P. M., Capizzi, A. M., ... Fletcher, J. M. (2006). The cognitive correlates of third-grade skill in arithmetic, algorithmic computation, and arithmetic word problems. Journal of Educational Psychology, 98(1), 29-43. doi: 10.1037/00220663.98.1.29

Fuchs, L. S., Geary, D. C., Compton, D. L., Fuchs, D., Hamlett, C. L., \& Bryant, J. D. (2010a). The contributions of numerosity and domain-general abilities to school readiness. Child Development, 81(5), 1520-1533. doi: 10.1111/j.14678624.2010.01489.x

Fuchs, L. S., Geary, D. C., Compton, D. L., Fuchs, D., Hamlett, C. L., Seethaler, P. M., ... Schatschneider, C. (2010b). Do different types of school mathematics development depend on different constellations of numerical versus general cognitive abilities? Developmental Psychology, 46(6), 1731-1746. doi: 10.1037/a0020662

Gilbert, J. K., \& Fuchs, L. S. (2017). Bivariate developmental relations between calculations and word problems: A latent change approach. Contemporary Educational Psychology, 51, 83-98. 
Ortiz, S. S., Sterpin, L. F., Barreyro, J. P. y Formoso, J. / RACC, 2021, Vol. 13, №2, 24-37

doi: 10.1016/j.cedpsych.2017.06.008

Geary, D. C., Hoard, M. K., Byrd-Craven, J., \& DeSoto, M. C. (2004). Strategy choices in simple and complex addition: Contributions of working memory and counting knowledge for children with mathematical disability. Journal of Experimental Child Psychology, 88(2), 121-151. doi: 10.1016/j.jecp.2004.03.002

Gomes-Koban, C., Simpson, I. C., Valle, A., \& Defior, S. (2017). Oral vocabulary training program for Spanish third-graders with low socio-economic status: A randomized controlled trial. PloS ONE, 12(11), e0188157. doi: 10.1371/journal.pone.0188157

Green, S., \& Higgins, J. (2006). Cochrane Handbook for Systematic Reviews of Interventions. Estados Unidos: Wiley.

Groen, G., \& Resnick, L. B. (1977). Can preschool children invent addition algorithms? Journal of Educational Psychology, 69(6), 645-652. doi: 10.1037/0022-0663.69.6.645

Hornburg, C. B., Schmitt, S. A., \& Purpura, D. J. (2018). Relations between preschoolers' mathematical language understanding and specific numeracy skills. Journal of Experimental Child Psychology, 176, 84-100. doi: 10.1016/j.jecp.2018.07.005

Inglis, M., \& Gilmore, C. (2014). Indexing the approximate number system. Acta Psychologica, 145, 147-155. doi: 10.1016/j.actpsy.2013.11.009

Johnson-Laird, P. N. (2006). How we reason. Oxford, Inglaterra: Oxford University Press.

Jordan, N. C., \& Hanich, L. B. (2000). Mathematical thinking in second-grade children with different forms of LD. Journal of Learning Disabilities, 33(6), 567-578. doi: 10.1177/002221940003300605

Jordan, N. C., Hanich, L. B., \& Kaplan, D. (2003). Arithmetic fact mastery in young children: A longitudinal investigation. Journal of Experimental Child Psychology, 85(2), 103-119. doi: 10.1016/S0022-0965(03)00032-8

Kaufman, A. S., \& Kaufman, N. L. (1983). Batería de evaluación de Kaufman para niños. Madrid, España: TEA Ediciones.

Kikas, E., Männamaa, M., Kumari, V., \& Ulst, T. (2008). The Relationships among Verbal Skills of Primary School Students with Specific Learning Disabilities and a Typically Developing Comparison Group. International Journal of Disability, Development and Education, 55(4), 315-329. doi: $10.1080 / 10349120802489521$

Kleemans, T., Segers, E., \& Verhoeven, L. (2018). Role of linguistic skills in fifth-grade mathematics. Journal of Experimental Child Psychology, 167, 404-413. doi: 10.1016/j.jecp.2017.11.012

Langan, D., Higgins, J. P. T., Jackson, D., Bowden, J., Veroniki, A. A., Kontopantelis, E., ... Simmonds, M. (2019). A comparison of heterogeneity variance estimators in simulated random-effects meta-analyses. Research Synthesis Methods, 10(1), 83-98. doi: 10.1002/jrsm.1316

LeFevre, J. A., Sadesky, G. S., \& Bisanz, J. (1996). Selection of procedures in mental addition: Reassessing the problem size effect in adults. Journal of Experimental Psychology: Learning Memory and Cognition, 22(1), 216-230. doi: 10.1037/0278-7393.22.1.216

Lonigan, C. J., Wagner, R. K., Torgesen J. K., \& Rashotte, C. A (2007). Test of Preschool Early Literacy (TOPEL). Austin, Texas: Pro-Ed.

Männamaa, M., Kikas, E., Peets, K., \& Palu, A. (2012). Cognitive correlates of math skills in third-grade students. Educational Psychology, 32(1), 21-44. doi: 10.1080/01443410.2011.621713

Mazzocco, M. M. M., Feigenson, L., \& Halberda, J. (2011). Impaired acuity of the approximate number system underlies mathematical learning disability (dyscalculia). Child Development, 82(4), 12241237. doi: 10.1111/j.1467-8624.2011.01608.x

Múñez, D., Orrantia, J., \& Rosales, J. (2013). The effect of external representations on compare word problems: Supporting mental model construction. The Journal of Experimental Education, 81(3), 337355. doi: 10.1080/00220973.2012.715095

Oakhill, J. V., \& Cain, K. (2012). The Precursors of Reading Ability in Young Readers: Evidence From a Four-Year Longitudinal Study. Scientific Studies of Reading, 16(2), 91-121. doi: $10.1080 / 10888438.2010 .529219$

Oakhill, J., Cain, K., \& McCarthy, D. (2015). Inference processing in children: the contributions of depth and breadth of vocabulary knowledge. En E. O'Brien, A. Cook, \& R. Lorch Jr. (Eds.), Inferences during Reading (pp. 140-159). Cambridge: Cambridge University. doi: 10.1017/cbo9781107279186.008

Odic, D., Libertus, M. E., Feigenson, L., \& Halberda, J. (2013). Developmental change in the acuity of approximate number and area representations. Developmental Psychology, 49(6), 1103-1112. doi: $10.1037 / \mathrm{a} 0029472$

Ostad, S. A. (2000). Cognitive subtraction in a developmental perspective: Accuracy, speed-ofprocessing and strategy-use differences in normal and mathematically disabled children. Focus on Learning Problems in Mathematics, 22(2), 18-32.

Park, J., \& Brannon, E. M. (2013). Training the approximate number system improves math proficiency. Psychological Science, 24(10), 20132019. doi: $10.1177 / 0956797613482944$

Perfetti, C., \& Hart, L. (2002). The lexical quality hypothesis. En L. Verhoeven, C. Elbro, \& P. Reitsma (Eds.), Precursors of Functional Literacy (pp.189-213). Amsterdam, Países Bajos: John Benjamin Publishing Co. doi: 
10.1075/swll.11.14per

Pina, V., Fuentes, L. J., Castillo, A., \& Diamantopoulou, S. (2014). Disentangling the effects of working memory, language, parental education, and nonverbal intelligence on children's mathematical abilities. Frontiers in Psychology, 5, 415. doi: 10.3389/fpsyg.2014.00415

Purpura, D. J., \& Ganley, C. M. (2014). Working memory and language: Skill-specific or domaingeneral relations to mathematics? Journal of Experimental Child Psychology, 122(1), 104-121. doi: 10.1016/j.jecp.2013.12.009

Purpura, D. J., Hume, L. E., Sims, D. M., \& Lonigan, C. J. (2011). Early literacy and early numeracy: The value of including early literacy skills in the prediction of numeracy development. Journal of Experimental Child Psychology, 110(4), 647-658. doi: 10.1016/j.jecp.2011.07.004

Sasanguie, D., Defever, E., Maertens, B., \& Reynvoet, B. (2014). The approximate number system is not predictive for symbolic number processing in kindergarteners. Quarterly Journal of Experimental Psychology (HOVE), 67(2), 271-280. doi: 10.1080/17470218.2013.803581

Sesma, H. W., Mahone, E. M., Levine, T., Eason, S. H., \& Cutting, L. E. (2009). The contribution of executive skills to reading comprehension. Child Neuropsychology, 15(3), 232-246. doi: 10.1080/09297040802220029

Seethaler, P. M., Fuchs, L. S., Fuchs, D., \& Compton, D. L. (2012). Predicting first graders' development of calculation versus word-problem performance: The role of dynamic assessment. Journal of Educational Psychology, 104(1), 224-234. doi: 10.1037/a0024968

Starr, A., Libertus, M. E., \& Brannon, E. M. (2013). Infants Show Ratio-dependent Number Discrimination Regardless of Set Size. Infancy, 18(6), 927-941. doi: 10.1111/infa.12008

Strasser, K., \& del Río, F. (2014). The Role of Comprehension Monitoring, Theory of Mind, and Vocabulary Depth in Predicting Story Comprehension and Recall of Kindergarten Children. Reading Research Quarterly, 49(2), 169187. doi: $10.1002 /$ rrq. 68

Thevenot, C. (2010). Arithmetic word problem solving: Evidence for the construction of a mental model. Acta Psychologica, 133(1), 90-95. doi: 10.1016/j.actpsy.2009.10.004

Thevenot, C. (2017). Arithmetic Word Problem Solving: The Role of Prior Knowledge. En D. C. Geary, D. B. Berch, R. J. Ochsendorf, \& K. M. Koepke (Eds.), Mathematical Cognition and Learning, Acquisition of Complex Arithmetic Skills and Higher-Order Mathematics Concepts (pp. 47-66). Cambridge, EEUU: Academic Press. doi: 10.1016/B978-0-12805086-6.00003-5
Veroniki, A. A., Jackson, D., Viechtbauer, W., Bender, R., Bowden, J., Knapp, G., ... Salanti, G. (2016). Methods to estimate the between-study variance and its uncertainty in meta-analysis. Research Synthesis Methods, 7(1), 55-79. doi: 10.1002/jrsm.1164

Von Hippel, P. T. (2015). The heterogeneity statistic I(2) can be biased in small meta-analyses. BMC Medical Research Methodology, 15, 35. doi: 10.1186/s12874-015-0024-z

Wagner, R. K., Torgesen, J. K., \& Rashotte, C. A. (1999). Comprehensive Test of Phonological Processing (CTOPP). Austin, Texas: Pro-Ed.

Wechsler, D. (1994). Test de inteligencia para niños WISC-III, Manual. Buenos Aires, Argentina: Paidós.

Wechsler, D. (1999). Wechsler Abbreviated Scales of Intelligence: WASI. San Antonio, Texas: Psychological Corporation.

Wechsler, D. (2005). Wechsler Individual Achievement Test-Second UK Edition. Oxford, England: Harcourt Assessment.

Wechsler, D. (2012). Test de inteligencia para niños WISC-IV. Buenos Aires, Argentina: Paidós.

Wiig, E. H., Secord, W., \& Semel, E. M. (2004). CELFPreschool 2: Clinical evaluation of language fundamentals-preschool. San Antonio, Texas: Pearson/Psychological Corporation.

Wilkinson, G. S. (1993). Wide range achievement 3. Wilmington, Delaware: Wide Range.

Woodcock, R. W. (1997). Woodcock Diagnostic Reading Battery. Itasca, IL: Riverside Publishing.

Woodcock, R. W., McGrew, K. S., \& Mather, N. (2001). Woodcock- Johnson III. Itasca, IL: Riverside Publishing. 